\title{
Preparation of Conductive PANI/PVA Composites via an Emulsion Route
}

\author{
Xiang-Qin Wang, ${ }^{1}$ Bin-Jie Xin, ${ }^{1}$ and Jian $\mathrm{Xu}^{2}$ \\ ${ }^{1}$ College of Fashion, Shanghai University of Engineering Science, Longteng Road, Shanghai 201620, China \\ ${ }^{2}$ Shangtex Holding Co., Ltd., Pingliang Road, Shanghai 200082, China
}

Correspondence should be addressed to Bin-Jie Xin; xinbj@sues.edu.cn

Received 12 July 2013; Accepted 6 November 2013

Academic Editor: Xiao-Hong Qin

Copyright (C) 2013 Xiang-Qin Wang et al. This is an open access article distributed under the Creative Commons Attribution License, which permits unrestricted use, distribution, and reproduction in any medium, provided the original work is properly cited.

\begin{abstract}
A facile and novel strategy for preparing polyaniline/polyvinyl alcohol (PANI/PVA) composite emulsion is reported wherein the reaction is carried out via the emulsion polymerization using ammonium peroxydisulfate (APS) as the oxidizing agent and dodecylbenzene sulfonic acid (DBSA) as the protonic acid. The PANI/PVA composite membranes have been characterized using optical microscope, scanning electron microscope (SEM), thermogravimetric analysis (TGA), Fourier transform infrared spectroscopy (FTIR), and electrochemical workstation. It is interesting that the electrical conductivity of the PANI/PVA composites is estimated to be as high as $1.28 \mathrm{~S} / \mathrm{cm}$. The experimental results show that the surface of PANI/PVA composite membranes exhibits good integrity. The PANI particles at the nanoscale are dispersed in the PVA matrix, and the electrochromic behaviors of PANI/PVA composites obtained at different polymerization temperatures can be compared based on cyclic voltammetry $(\mathrm{CV})$ curves, revealing that PANI/PVA composites synthesized at room temperature are better than those synthesized at low temperature.
\end{abstract}

\section{Instruction}

Polyaniline (PANI), one of the most thoroughly studied conducting polymers, has received great attention due to its high electrical conductivity, easy producibility, thermal and environmental stability, energy storage, and sensible and electrochemical properties, as well as relatively low cost [1]. The potential applications of PANI include organic lightweight batteries [2], redox capacitors, electromagnetic shielding devices [3], microelectronic devices [4,5], sensors [6-11], and electrochromic displays [12-19]. A method of preparing the PANI was first presented by Letheby in 1862 [20]; however, the conductive nature and electrochemical property have not been studied in-depth until the late last century. From the early 20th century on, occasional reports about the structure of PANI were published. Subsequent to the investigation of other highly conductive organic materials, MacDiarmid demonstrated the conductive states of PANI which arose upon protonic doping of the emeraldine form of PANI [21]. Conductive polymers such as PANI remain of widespread interest [22], providing an opportunity to address fundamental issues of importance to condensed matter physics, including the metal insulator transition [23], the Peierls instability, and quantum decoherence [24].

During the past few decades, attention has been devoted to the use of conductive polymeric composites for fabrication of displays and sensor devices due to their better stability, flexibility, and cost effectiveness [25]. Extensive studies on the gas sensing properties of conducting PANI composites [26, 27] and polypyrrole (PPy) composites for various oxidizing and reducing gases have been reported [28, 29]. However, in order to solve the limitations on their solubility and electrochemical properties of the conductive PANI composites, which are mainly due to the polymerization ways, nonhomogeneous composite formation, and doping-dedoping properties of the conducting polymers, it is necessary to develop a real composite of conductive polymers with the essential properties of the conductive polymers in addition to the improved processibility and mechanical properties.

In this work, an attempt has been made to prepare a conducting PANI composite material with reasonable conductivity along with good mechanical properties. The PVA reagent 
is selected as the polymer matrix for the preparation of the composite membrane, and the emulsion polymerization technique with the DBSA as proton acid dopant is used to synthesize the PANI/PVA emulsion. The properties of the PANI/PVA composites obtained from emulsion polymerization have been characterized attached with the related explanations and discussions.

\section{Experimental}

2.1. Raw Materials and Reagents. Aniline (AN), polyvinyl alcohol (PVA), ammonium persulfate (APS), dodecyl-benzene sulfonic acid (DBSA), and indium-tin-oxide (ITO, (35 \pm $5 \mathrm{ohm} / \mathrm{sq})$ ) film were purchased. All the chemical reagents were used without further purification, and the distilled water was used during the experiments.

\subsection{Preparation of PANI/PVA Composite Emulsion. PANI/} PVA composites emulsion was produced using the APS oxidant dissolved in emulsion contained PVA, DBSA, and AN monomer in a three-neck round bottomed flask equipped with mechanical stirring, thermometer, and titration funnel. At first, the the solution contained in the flask was heated at $90^{\circ} \mathrm{C}$ for 20 minutes to 40 minutes to make PVA particles dissolve adequately, and the reaction mixture was stirred for 30 minutes to ensure that the mixture was cooled to room temperature. The solution appeared to be milk white after the addition of the DBSA reagent and AN monomer sequently. The $10 \mathrm{~mL}$ aqueous solution of APS was dropped into the emulsion at different temperatures. The stirring of the mixture continued for another 4 hours after the color of emulsion turned into dark green; the PANI/PVA composite emulsion was obtained for the preparation of the PANI/PVA composite membranes.

2.3. Preparation of PANI/PVA Composite Membranes. PANI/ PVA composite membranes were prepared using the spincoating machine (KW-4A Model). An appropriate amount of solution was spread on the substrate of the instrument into a thin film at the rotational speed of $600 \mathrm{rpm}$ (evolutions per minute) for $15 \mathrm{~s}$ (second) and $5000 \mathrm{rpm}$ for $40 \mathrm{~s}$. The PANI/PVA composite membranes were obtained after drying in a vacuum oven at the room temperature for about 4 hours.

2.4. Characterization and Analysis. The four-probe tester with a measuring range of $10^{-2} \sim 10^{5} \Omega$ and the UT70A universal digital multimeter with a higher limit of the measuring range about $10^{3} \Omega$ were used to measure the surface resistivity of PANI/PVA composite membranes.

The XS-213 optical microscope and the S-3400N scanning electron microscope (SEM) with a resolution at nanoscale were used to analyze the morphology of the PANI/PVA composite membranes.

The AVATAR 380 Fourier transform infrared spectroscopy (FTIR) analyzer was used to characterize the molecular structure and chemical composition of PANI/PVA composites. The spectra data were recorded from 3800 to $750 \mathrm{~cm}^{-1}$ with the step size of about $1.929 \mathrm{~cm}^{-1}$.
The Germany STA PT-1000 thermal gravimetric (TG) analyzer was used to investigate the thermal stability of PANI/PVA composites. The samples were heated from room temperature to $700^{\circ} \mathrm{C}$ at a slow heating rate of $10^{\circ} \mathrm{C} / \mathrm{min}$. The TG experiment was carried out in the nitrogen atmosphere with the gas flow of $100 \mathrm{~mL} / \mathrm{min}$.

The CHI420A type electrochemical workstation was used to investigate the electrochromic properties of PANI/PVA composite membranes.

\section{Results and Discussion}

3.1. The Doping Mechanism of PANI. The PANI was synthesized with the molar ratio of APS to aniline monomer set to be $0.95-1: 1$ and the molar ratio of DBSA to aniline monomer set to be $1.03: 1$ in our experiments. The conductivity of PANI composite membrane can be up to $1.28 \mathrm{~S} / \mathrm{cm}$.

As illustrated in Figure 1, the basic skeleton of the molecular chain of PANI does not change after doping by DBSA protonic acid; only the electrons are transferred and rearranged within the molecular chain. The doping process of PANI could be divided into three steps from nonconductive state to a conductive state.

Step 1. DBSA is decomposed into $\mathrm{H}^{+}$in the emulsion, and then the $\mathrm{H}^{+}$is combined with $\mathrm{N}$ atom, which is from the polymer chain between the head and the tail of the class quinone of PANI oxidation unit. The univalent $\mathrm{N}$ atoms were converted to univalent positive charge, because the $\mathrm{H}^{+}$had no valence electrons; when bonding with the $\mathrm{N}$, the $\mathrm{N}$ atoms provided the electron, thereby enabling the polymer chains to take positive charge.

Step 2. Because the class quinone structure with univalent $\mathrm{N}$ atoms is unstable, the rearrangement of electrophilic substitution reaction is prone to occur in AN molecular. So, the polymer chains within the charge can be redistributed, and the partial positive charge $\mathrm{N}$ atoms are transferred from the quinone structure to the $\mathrm{N}$ atoms on the benzene's reducing unit. On the contrary, the electrons can be diverted from the benzene structure to the quinone structure; the benzene structure is partially oxidized attributed to the loss of electrons, and the quinone ring of the quinone structure is partially reduced due to the achieving of electrons. So, the ring electronic structure tends to be similar. Therefore, it can be seen that the doping of PANI is a unique intramolecular redox reaction, no gain or loss of electrons.

Step 3. The charge rearrangement of the doped PANI became similar or even identical electronic structure; ultimately, the formation of a large conjugated system was obtained throughout the whole molecular chain; the positive charge was nonuniformly distributed in the entire conjugated system. The charge delocalization of the molecular chain of PANI intramolecular and intermolecular was optimized to promote the moving of charges in the molecular chain due to using a large scale and slow diffusion of the organic acid DBSA.

Simultaneously, the process of PANI hydrolysis can be promoted by the solvent effects between dodecylbenzene 


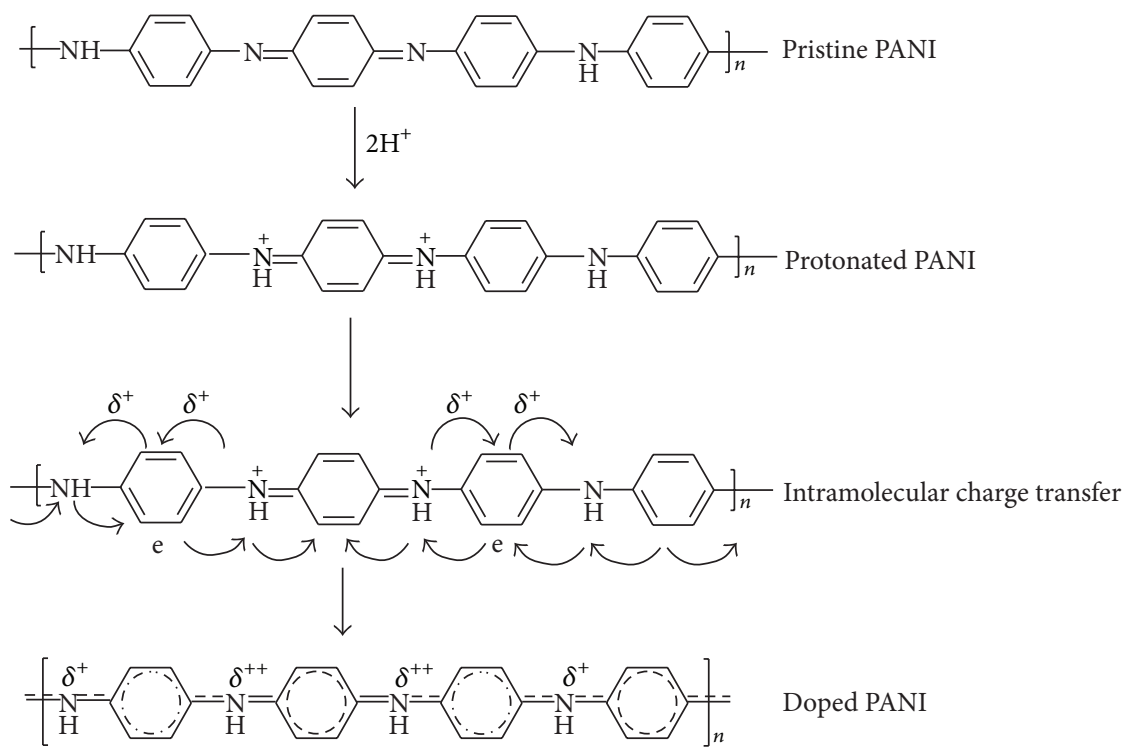

FIgURE 1: The doping mechanism of PANI.

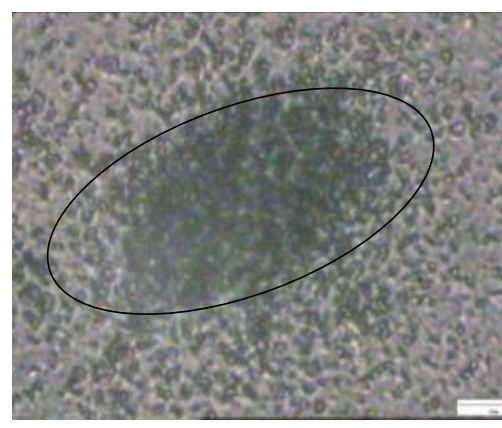

(a)

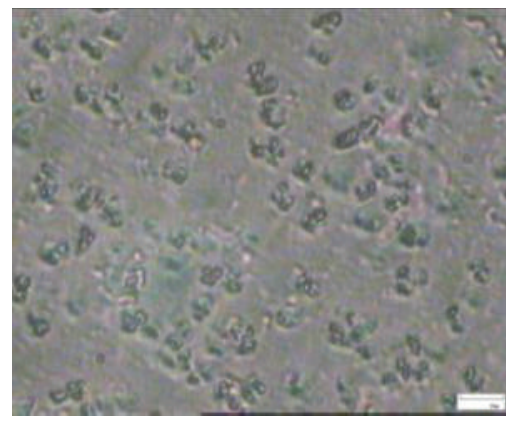

(b)

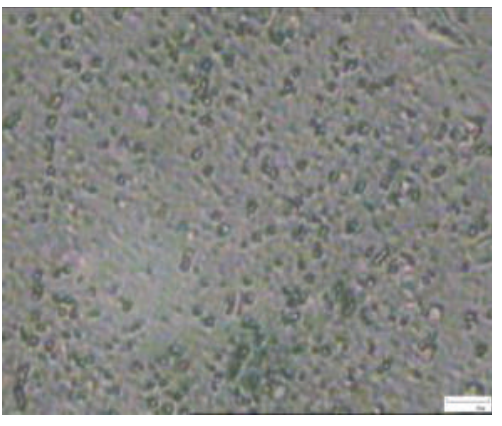

(c)

Figure 2: The optical microscope images of PANI/PVA composite membranes with the stirring speed of (a) $1000 \mathrm{rpm}$; (b) $1600 \mathrm{rpm}$; and (c) $2200 \mathrm{rpm}$.

sulfonic group and water; the good conductivity and improved solubility of the doped PANI can be achieved.

3.2. Morphology Analysis. The membrane samples were obtained via spin-coating method using the PANI/PVA emulsion synthesized in our laboratory; the stirring speed during the process of polymerizing varied between 1000, 1600 , and $2200 \mathrm{rpm}$, respectively.

Figures 2(a), 2(b), and 2(c) were the images of PANI captured by the optical microscope with a magnification of 3000x. The spherical shaped granules can be observed on the surface of the PVA polymer, with the diameter ranging from 0.5 to $1 \mu \mathrm{m}$. It could be seen that the low speed led to agglomeration. However, the dispersion of PANI particles could be optimized when the stirring speed increased to $2200 \mathrm{rpm}$.

Figure 3 was the SEM image captured by S-2700 SEM; the morphology of the PANI/PVA composite membrane was observed with a very excellent uniformity; the reason behind this is that the small force of PANI chains is insufficient to

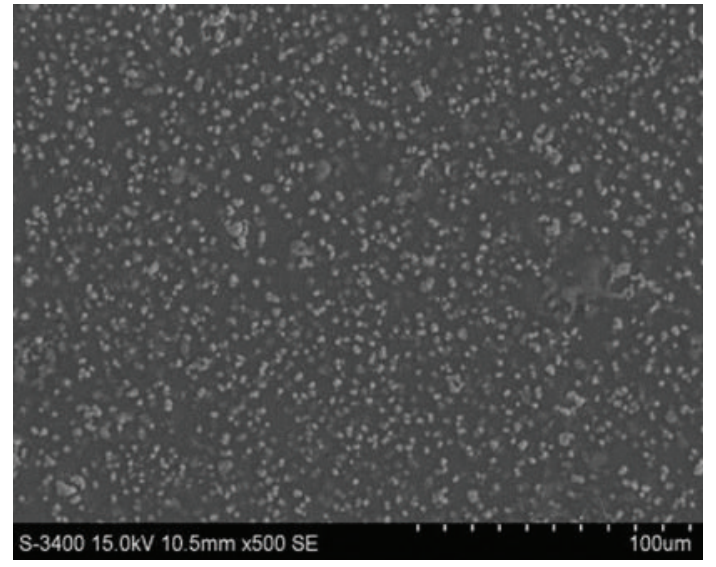

FIgURE 3: The SEM image of PANI/PVA composite membrane.

provide the minimum molecular chain force of agglomeration. Therefore, the PANI/PVA composite membrane should 


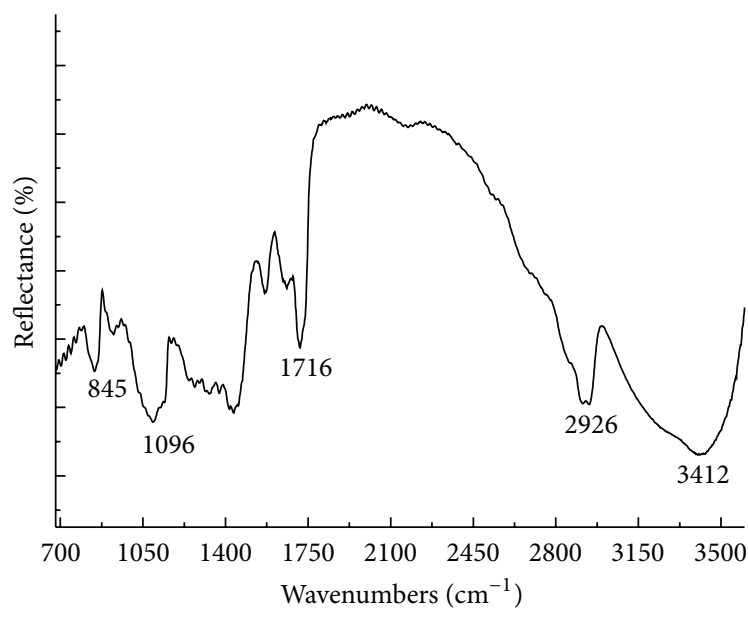

(a) PVA

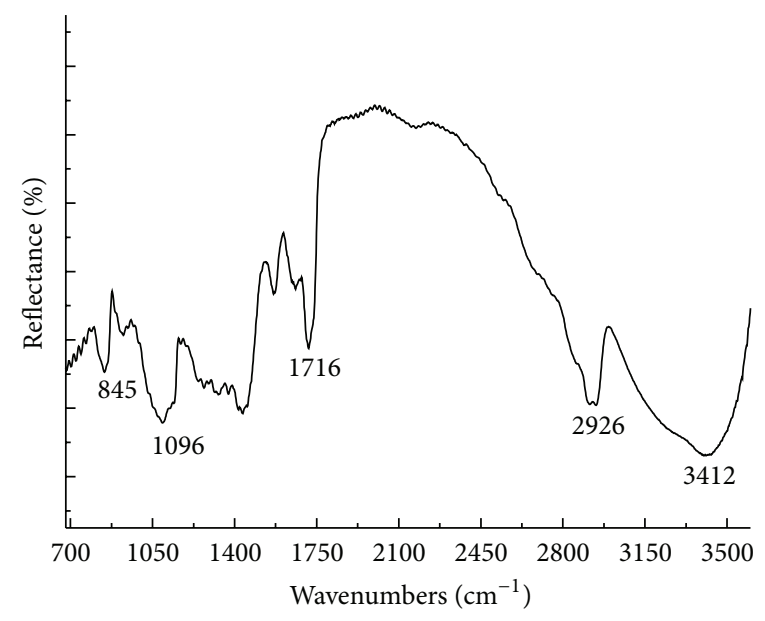

(b) PANI/PVA

FIGURE 4: The FTIR absorption spectrum of PVA (a) and PANI/PVA (b).

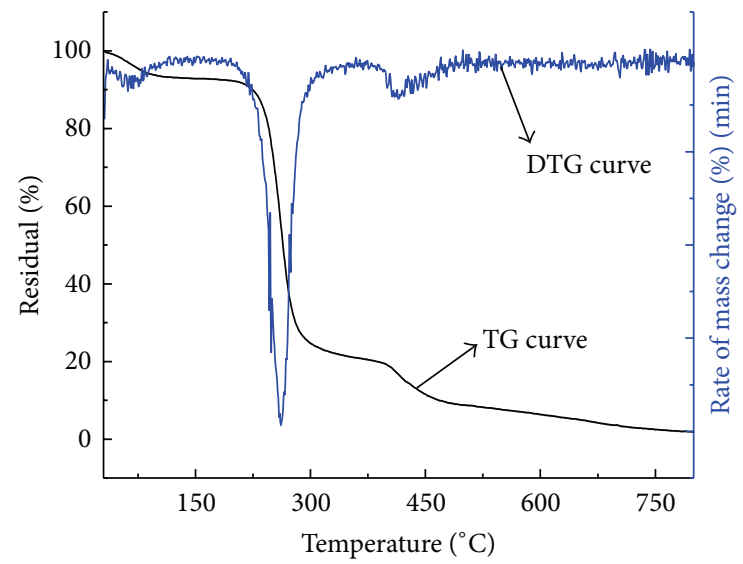

(a) PVA

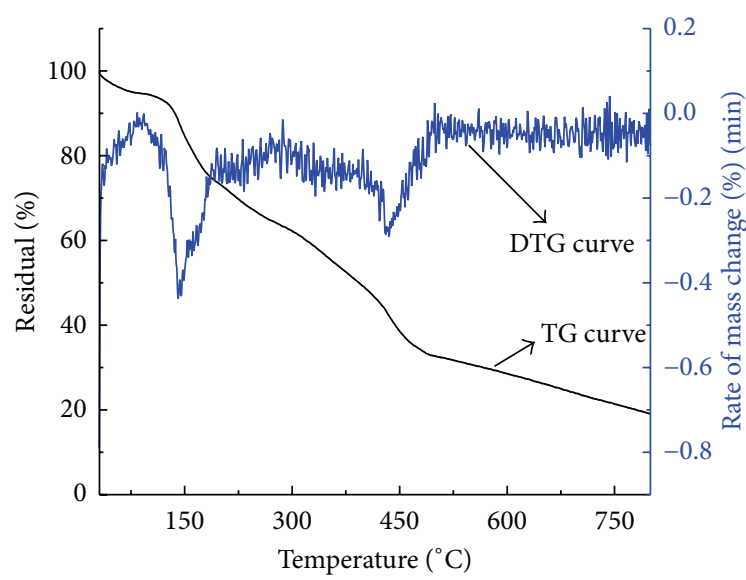

(b) PANI/PVA

FIGURE 5: The curve of PANI/PVA composite membrane about thermal gravimetric analysis.

be suitable for the preparation of the electrospun material and the electrochromic devices.

3.3. The FT-IR Spectrum Analysis. The presence of pristine PVA was seen as strong absorption peaks at a wavenumber of $3412 \mathrm{~cm}^{-1}$, which was attributed to the $-\mathrm{OH}$ stretching bend, for the C-O stretch at $1096 \mathrm{~cm}^{-1}$ which was observed in Figure 4(a). In PANI/PVA composites, the peaks of PANI at $1699 \mathrm{~cm}^{-1}$ and $1456 \mathrm{~cm}^{-1}$ were attributed to the $\mathrm{C}=\mathrm{N}$ stretching vibration of quinone ring and the $\mathrm{C}=\mathrm{C}$ stretching vibration associated with quinone ring and benzene ring structures, respectively. The bands at $1402 \mathrm{~cm}^{-1}, 1165 \mathrm{~cm}^{-1}$ corresponding to the $\mathrm{C}-\mathrm{N}$ stretching vibration and the $\mathrm{C}-\mathrm{H}$ plane blending vibration were clearly seen. The $\mathrm{N}=\mathrm{Q}=\mathrm{N}$ class electronic absorption structure model stretching vibration absorption peak was at about $1058 \mathrm{~cm}^{-1}$ and $1112 \mathrm{~cm}^{-1}$ nearby, and the peak at $750 \sim 850 \mathrm{~cm}^{-1}$ was assigned to the $\mathrm{C}-\mathrm{H}$ plane bending vibration peak in 1,4 substituted benzene ring. The data analysis and the literature elaborated consensus [30].
The FTIR spectra of pure PVA and PANI/PVA were compared with the membrane samples from Figure 4; the spectrum of PVA was similar to that of PVA in the PANI/PVA composites; the presence of PANI in PANI/PVA composites had a little influence on the PVA.

3.4. The Thermogravimetric Analysis. Figure 5 showed the thermogravimetric curves (TGA) of pure PVA and PANI/ PVA composites at $10^{\circ} \mathrm{C} / \mathrm{min}$ heating rate. From the TGDTG curve, the thermal decomposition point presented obviously at the temperature of $260^{\circ} \mathrm{C}$, but from the thermal decomposition process of PANI/PVA composites, the typical decomposition point of PVA disappeared, and because PANI/PVA composite material contains an emulsifier called PVA, unreacted reagents called DBSA, the thermal decomposition process of PANI/PVA material was not obvious, and the whole curve still showed a decreasing trend. And the PANI/PVA composites seemed to undergo four stages of degradation based on Figure 5 . 


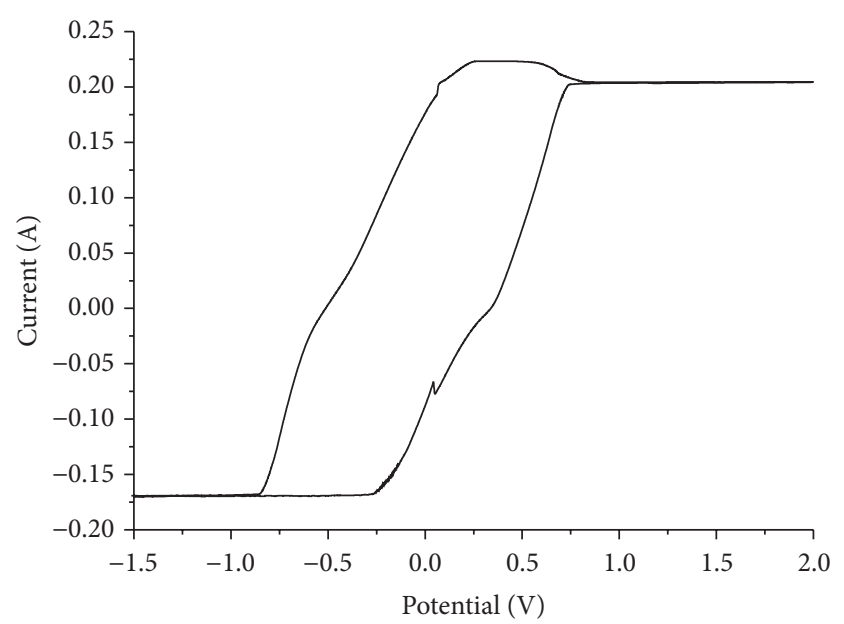

(a) At room temperature

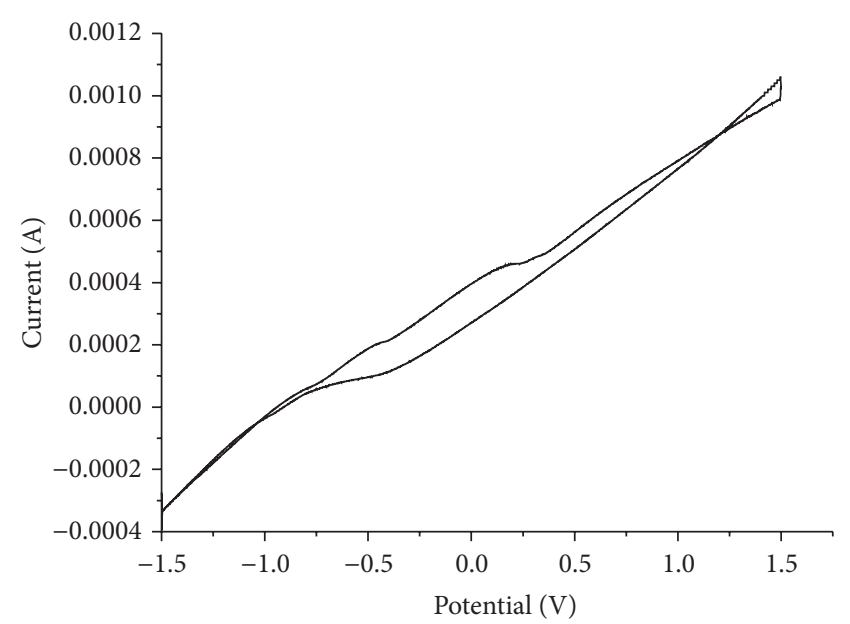

(b) In ice-water bath

Figure 6: The CV curves of PANI/PVA composite membranes.

The first part was the water-based weight loss stage. The evaporation of deionized water and the bonding water from the intermolecular led to the mass loss of composite materials when the temperature was up to $100^{\circ} \mathrm{C}$, and the volatilization of a small amount of additives might be the part of weightlessness.

The second part was weight loss phase of PVA thermal decomposition. As the temperature continuously increased to $140-180^{\circ} \mathrm{C}$, PVA dehydration etherification played an important role in weight loss; continuing to heat up, PANI also lost a small amount of weight due to PANI oxidation.

The third part was mainly the section of PANI thermal decomposition. As the temperature increased to $273^{\circ} \mathrm{C}$, the increasing movement rate of polymer macromolecular chains was enhanced as the temperature increased to $273^{\circ} \mathrm{C}$. Meanwhile, the molecules released gases leading to the mass loss. Through the bond energy analysis, the production of gases such as $\mathrm{NH}_{3}$ and $\mathrm{CO}_{2}$ was the main mass loss at this stage.

The fourth stage was called carbonization phase; the temperature rose to $560^{\circ} \mathrm{C}$, and the TG curve decreased slowly and then reached a platform. At this stage, most of polymers were carbonized and the temperature had less impact on the mass loss of the residues. As depicted in Figure 5, the residual mass tended to a steady state.

3.5. Cyclic Voltammetry Curves for the PANI/PVA Composites. Conductive PANI membrane samples deposited on the transparent conducting ITO electrodes with the coating thickness of $20 \mu \mathrm{m}$ were prepared by the KW-4A spin coater. At a constant rate of $200 \mathrm{mV} / \mathrm{S}$ from the initial potential of $-1.5 \mathrm{~V}$ to the final potential of $+1.5 \mathrm{~V}$ using the hydrochloric acid $(1 \mathrm{Mol} / \mathrm{L})$ as electrolyte, the CV curves for PANI/PVA composites, obtained under room temperature and icewater bath separately, were carried out for electrochemical characterization of the PANI/PVA composite membranes, and the results were shown in Figure 6.

With applied potentials from -1.5 to $+1.5 \mathrm{~V}$, both devices showed electrochromic activity with color change from yellowish-green (reduction state) to green (neutral state) and sky blue (oxidation state). Comparing the CVs in Figure 6, it could be found that PANI/PVA composite membranes have unobvious redox peaks, and the area of PANI/PVA composite membrane at room temperature surrounded by the CV curve is larger than that at ice-water bath, indicating that room temperature condition has much more specific electrochemical property.

\section{Result and Prospect}

The PANI/PVA composite was prepared via emulsion polymerization followed by the proton acid doping. The proposed polymerization method indicated that emulsion polymerization was an effective and facile strategy for the fabrication of great amount of PANI/PVA composites. PANI particles with estimated diameter of $0.5 \sim 1 \mu \mathrm{m}$ were shown to be evenly dispersed in PVA matrix. The typical $\mathrm{C}=\mathrm{N}$ stretching vibration of the quinone ring at $1699 \mathrm{~cm}^{-1}$ and the $\mathrm{C}=\mathrm{C}$ stretching vibration associated with the quinone ring and the benzene ring structures at $1456 \mathrm{~cm}^{-1}$ were the characteristic peaks of conductive PANI. The PANI/PVA composites thermal decomposition was up to $273^{\circ} \mathrm{C}$. The $\mathrm{CV}$ curves indicated that the PANI/PVA material at room temperature has much more specific electrochemical property and was much more appropriate for electrochromic device than that at low temperature.

Further work is on the way to improve the thermal stability and measure the conductivity change of the PANI composite membranes under different potentials in order to make a more comprehensive understanding of the mechanism of the PANI composite electrochromic device.

\section{Acknowledgment}

This work was financially supported by Shanghai University of Engineering Science Graduate Student Research Innovation Project (2012yjs44). 


\section{References}

[1] N. Gospodinova and L. Terlemezyan, "Conducting polymers prepared by oxidative polymerization: polyaniline," Progress in Polymer Science, vol. 23, no. 8, pp. 1443-1484, 1998.

[2] M. Kaneko and H. Nakamura, "Photoresponse of a liquid junction polyaniline film," Journal of the Chemical Society, Chemical Communications, no. 6, pp. 346-347, 1985.

[3] C. Y. Lee, H. G. Song, K. S. Jang, E. J. Oh, A. J. Epstein, and J. Joo, "Electromagnetic interference shielding efficiency of polyaniline mixtures and multilayer films," Synthetic Metals, vol. 102, no. 1-3, pp. 1346-1349, 1999.

[4] E. W. Paul, A. J. Ricco, and M. S. Wrighton, "Resistance of polyaniline films as a function of electrochemical potential and the fabrication of polyaniline-based microelectronic devices," Journal of Physical Chemistry, vol. 89, no. 8, pp. 1441-1447, 1985.

[5] S. A. Chen and Y. Fang, "Polyaniline schottky barrier: effect of doping on rectification and photovoltaic characteristics," Synthetic Metals, vol. 60, no. 3, pp. 215-222, 1993.

[6] C. H. Liu, K. T. Liao, and H. J. Huang, "Amperometric immunosensors based on protein A coupled polyanilineperfluorosulfonated ionomer composite electrodes," Analytical Chemistry, vol. 72, no. 13, pp. 2925-2929, 2000.

[7] V. Svetlicic, L. L. Miller, and A. J. Schmidt, "Conductometric sensors based on the hypersensitive response of plasticized polyaniline films to organic vapors," Chemistry of Materials, vol. 10, no. 11, pp. 3305-3307, 1998.

[8] H. Sangodkar, S. Sukeerthi, R. S. Srinivasa, R. Lal, and A. Q. Contractor, "A biosensor array based on polyaniline," Analytical Chemistry, vol. 68, no. 5, pp. 779-783, 1996.

[9] S. Sukeerthi and A. Q. Contractor, "Molecular sensors and sensor arrays based on polyaniline microtubules," Analytical Chemistry, vol. 71, no. 11, pp. 2231-2236, 1999.

[10] C. Basavaraja, W. J. Kim, D. G. Kim, and D. S. Huh, "Behavior of polyaniline-dodecylbenzene sulfonic acid/reduced graphene oxide nanocomposite films," Polymer Composites, vol. 33, no. 3, pp. 388-396, 2012.

[11] J. Wilson, S. Radhakrishnan, C. Sumathi, and V. Dharuman, "Polypyrrole polyaniline Au (PPy-PANi-Au) nanocomposite films for label-free electrochemical DNA sensing," Sensors and Actuators B, vol. 171-172, pp. 216-222, 2012.

[12] A. Kitani, J. Yano, and K. Sasaki, "ECD materials for the three primary colors developed by polyanilines," Journal of Electroanalytical Chemistry, vol. 209, no. 1, pp. 227-232, 1986.

[13] R. J. Mortimer, A. L. Dyer, and J. R. Reynolds, "Electrochromic organic and polymeric materials for display applications," Displays, vol. 27, no. 1, pp. 2-18, 2006.

[14] P. Somani, A. B. Mandale, and S. Radhakrishnan, "Study and development of conducting polymer-based electrochromic display devices," Acta Materialia, vol. 48, no. 11, pp. 2859-2871, 2000.

[15] C. Y. Xu, "The research present and the development trend of switchable window," in Proceedings of the International Textile Science and Technology Forum, pp. 35-40, 2010.

[16] P. R. Somani and S. Radhakrishnan, "Electrochromic materials and devices: present and future," Materials Chemistry and Physics, vol. 77, no. 1, pp. 117-133, 2003.

[17] Y. J. Ding, M. A. Invernale, D. M. D. Mamangun, A. Kumar, and G. A. Sotzing, "A simple, low waste and versatile procedure to make polymer electrochromic devices," Journal of Materials Chemistry, vol. 21, no. 32, pp. 11873-11878, 2011.
[18] M. A. Invernale, Y. J. Ding, and G. A. Sotzing, "The effects of coloured base fabric on electrochromic textile," Coloration Technology, vol. 127, no. 3, pp. 167-172, 2011.

[19] J. Luo, Y. Ma, J. Pei, and Y. Song, "Recent progress on organic and polymeric electrochromic materials," Current Physical Chemistry, vol. 1, no. 3, pp. 216-231, 2011.

[20] H. J. Letheby, "On the production of a blue substance by the electrolysis of sulphate of aniline," Journal of the Chemical Society, vol. 15, pp. 161-163, 1862.

[21] J. Chiang and A. G. MacDiarmid, “Polyaniline’: protonic acid doping of the emeraldine form to the metallic regime," Synthetic Metals, vol. 13, no. 1-3, pp. 193-205, 1986.

[22] A. J. Heeger, "Semiconducting and metallic polymers: the fourth generation of polymeric materials (Nobel lecture)," Angewandte Chemie-International Edition, vol. 40, no. 14, pp. 2591-2611, 2001.

[23] G. Tzamalis, N. A. Zaidi, and A. P. Monkman, "Applicability of the localization-interaction model to magnetoconductivity studies of polyaniline films at the metal-insulator boundary," Physical Review B, vol. 68, no. 24, Article ID 245106, 11 pages, 2003.

[24] C. J. Cattena, R. A. Bustos-Marún, and H. M. Pastawski, "Crucial role of decoherence for electronic transport in molecular wires: polyaniline as a case study," Physical Review B, vol. 82, no. 14, Article ID 144201, 10 pages, 2010.

[25] S. J. Su and N. Kuramoto, "Synthesis of processable polyaniline complexed with anionic surfactant and its conducting blends in aqueous and organic system," Synthetic Metals, vol. 108, no. 2, pp. 121-126, 2000.

[26] P. P. Sengupta, S. Barik, and B. Adhikari, "Polyaniline as a gassensor material," Materials and Manufacturing Processes, vol. 21, no. 3, pp. 263-270, 2006.

[27] S. Dogan, U. Akbulut, T. Yalcin, S. Suzer, and L. Toppare, "Conducting polymers of aniline II. A composite as a gas sensor," Synthetic Metals, vol. 60, no. 1, pp. 27-30, 1993.

[28] J. Wang, S. Chen, and M. S. Lin, "Use of different electropolymerization conditions for controlling the size-exclusion selectivity at polyaniline, polypyrrole and polyphenol films," Journal of Electroanalytical Chemistry, vol. 273, no. 1-2, pp. 231242, 1989.

[29] A. Riul Jr., A. M. G. Soto, S. V. Mello, S. Bone, D. M. Taylor, and L. H. C. Mattoso, "An electronic tongue using polypyrrole and polyaniline," Synthetic Metals, vol. 132, no. 2, pp. 109-116, 2003.

[30] H. Gomez, M. K. Ram, F. Alvi, E. Stefanakos, and A. Kumar, "Novel synthesis, characterization, and corrosion inhibition properties of nanodiamond-polyaniline films," Journal of Physical Chemistry C, vol. 114, no. 44, pp. 18797-18804, 2010. 

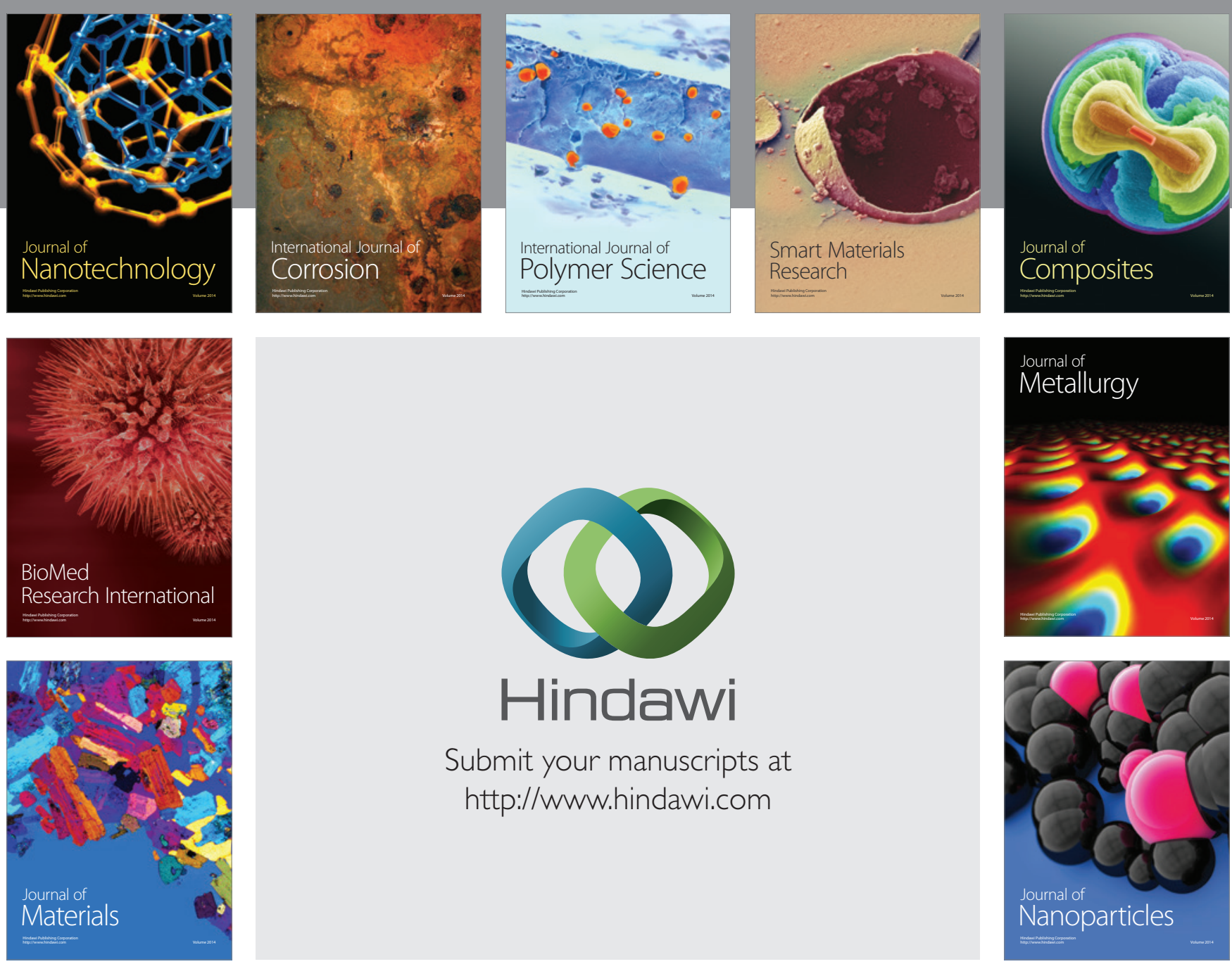

Submit your manuscripts at http://www.hindawi.com
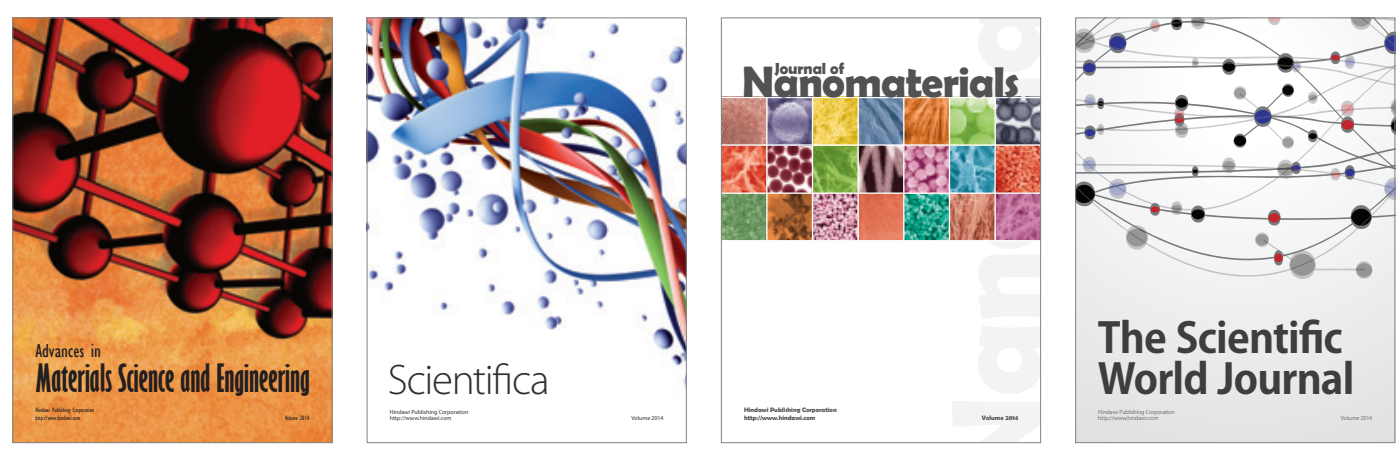

\section{The Scientific World Journal}
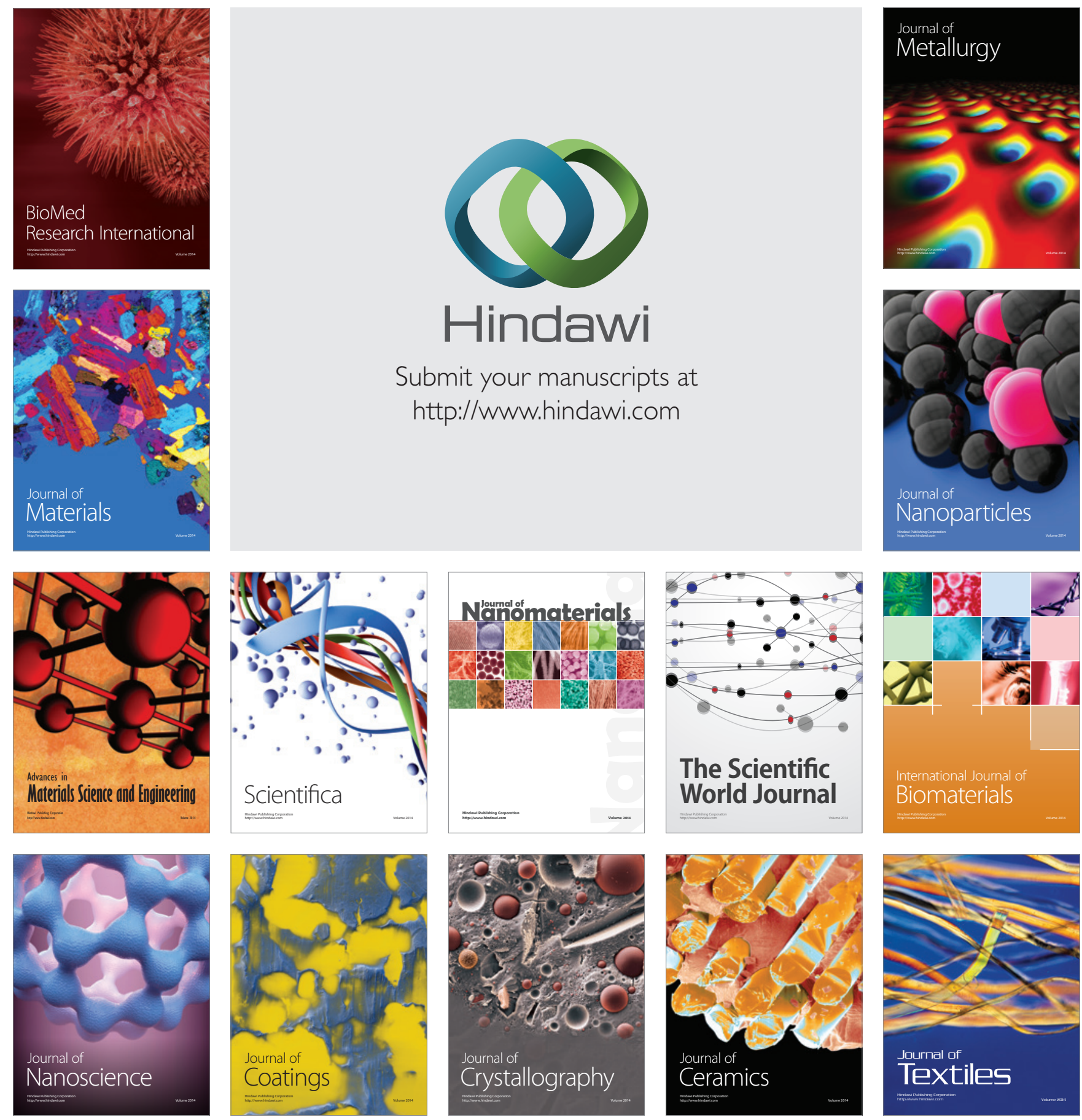